\title{
P950 Tissue cortisol vs lipolysis in ICU patients
}

loannis Ilias [1], Dimitra Vassiliadi [2], Maria Theodorakopoulou [2], Nikitas Nikitas [2], Marinela Tzanela [3], Sofia Apollonatou [2], Stylianos Tsagarakis [3], loanna Dimopoulou [2]

[1] Endocrine Unit, Elena Venizelou Hospital, Athens, Greece, [2] Second Department of Intensive Care, University of Athens Medical School, Attikon Hospital, Athens, Greece, [3]. Endocrine Department, Evangelismos Hospital, Athens, Greece

\section{Introduction}

The interplay of cortisol (F) and adipose tissue is complex and in many aspects is still obscure [1]. Plasma $\mathrm{F}$ has been shown to be positively associated with lipolysis [2].

\section{Aim}

To study in adipose tissue indices of lipolysis vs tissue $F$ with microdialysis (MD).

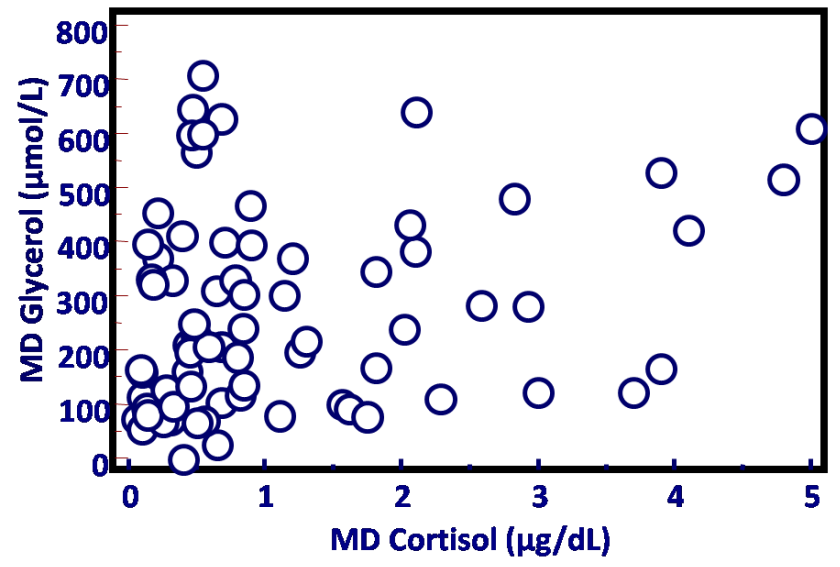

\section{Discussion}

We verified the well-known association (though modestly so) between lipolysis and $F$ (and in particular with interstitial/tissue levels of it) [2]. Changes in interstitial/ tissue $F$ may not be reflected in plasma (total) concentrations $[6,7]$. Thus it is interesting that we observed an - albeit weak association between tissue lipolysis (via MD GLYC levels) and MD F.

\section{Subjects and methods}

We studied 46 mechanically ventilated patients with a diagnosis of septic or nonseptic shock (Sho), systemic inflammatory response syndrome (SIRS) or severe sepsis (SSe) [3]. Upon ICU admission a MD catheter was inserted under sterile conditions into the subcutaneous adipose tissue of the upper thigh. Excluding patients on steroid therapy, on day $2(n=26)$, day $3(n=24)$ and day $4(n=22)$ MD samples were collected six times per day for MD glycerol (MD GLYC; used as an index of lipolysis) and tissue $F$. The mean of these 6 collections was used for analysis (normal values for adipose tissue GLYC glycerol < $200 \mu \mathrm{mol} / \mathrm{L}[4,5])$. Statistics were done with Spearman's rank correlation.

\section{Results}

Most samplings (44/72) indicated accentuated lipolysis with above-normal MD GLYC levels. MD GLYC was weakly correlated to MD F ( $r h o=0.246, p=0.038$ ).

\section{References}

[1]. Gathercole L.L., et al . (2011) PLOS ONE; 6: e26223.

[2].Djurhuus C.B., et al . (2002) Am J Physiol Endocrinol Metab; 283: E172-E177.

[3]. Levy M.M., et al . (2003) Crit Care Med 31: 1250-1256.

[4].Dimopoulou l., et al. (2011) Kinetics of adipose tissue microdialysis-derived metabolites iShock; 35: 343-348.

[5]. Ungerstedt U., Rostami E.(2004) Curr Pharm Des; 10: 2145 2152.

[6]. Cohen J., Venkatesh B.(2009) Crit Care Resusc; 11: 287-289.

[7]. Vassiliadi D.A., ET AL. (2013) J Crit Care; 28: 158-165. 